\title{
Aprendizaje emocional y de valores en la formación universitaria, aplicado al grado de Comunicación Audiovisual de la Universidad de Barcelona
}

\author{
Values and emotional learning in university training, \\ applied to the degree in Audiovisual Communication \\ at the University of Barcelona
}

Jorge Franganillo. Universidad de Barcelona. España.

franganillo@ub.edu

$[\mathrm{CV}]$ (iD) $\mathrm{G}$

Lydia Sánchez. Universidad de Barcelona. España.

lsanchezg@ub.edu

[CV] (iD) G

María Ángeles García Asensio. Universidad de Barcelona. España.

garciaasensio@ub.edu

$[\mathrm{CV}]$ (D) $\mathrm{G}$

Anna Marquès. Universidad de Barcelona. España.

anna.marques@ub.edu

[CV] (D)

Cómo citar este artículo / Referencia normalizada

Franganillo, J., Sánchez, L., García Asensio, M. A. y Marquès, A. (2021). Aprendizaje emocional y de valores en la formación universitaria, aplicado al grado de Comunicación Audiovisual de la Universidad de Barcelona. Revista Latina de Comunicación Social, 79, 151-173. https://doi.org/10.4185/RLCS-2021-1493

\section{RESUMEN.}

Este trabajo caracteriza el aprendizaje servicio (ApS), desde la perspectiva de la psicología positiva, como un modelo de aprendizaje capaz de articular la formación emocional y de valores en el ámbito de la Comunicación Audiovisual. Después, describe la experiencia que el Grupo de innovación docente consolidado en Comunicación y Medios Audiovisuales (In-COMAV), formado por un equipo docente multidisciplinar, desarrolla desde 2016 en el grado de Comunicación Audiovisual de la Universidad de Barcelona. A continuación, presenta, en el marco de esta experiencia, los criterios de viabilidad establecidos para la aceptación de proyectos de colaboración, los factores críticos de éxito y los resultados que la iniciativa ha tenido sobre el alumnado, el profesorado, la institución y la sociedad. Por último, señala una serie de desafíos y limitaciones que se deben superar para garantizar la viabilidad y la eficacia de los proyectos ApS en la enseñanza superior. 
PALABRAS CLAVE: Aprendizaje servicio; educación emocional; educación en valores; aprendizaje basado en proyectos; competencias profesionalizadoras; trabajo interdisciplinario.

\begin{abstract}
This work characterizes service learning (SL), from the perspective of positive psychology, as a learning model capable of articulating emotional and values learning in the field of Audiovisual Communication. Then, it describes the experience that the consolidated teaching innovation Group in Communication and Audiovisual Media (In-COMAV), made up of a multidisciplinary teaching team, has been developing since 2016 in the Audiovisual Communication degree at the University of Barcelona. It then presents, within the framework of this experience, the viability criteria established for the acceptance of collaboration projects, the critical success factors, and the results that the initiative has had on students, teachers, the institution, and society. Finally, it points out a series of challenges and limitations that must be overcome to guarantee the viability and effectiveness of SL projects in higher education.
\end{abstract}

KEYWORDS: Service-learning; emotional education; education in values; project-based learning; professionalizing skills; interdisciplinary work.

CONTENIDO: 1. Introducción. 2. Hipótesis y objetivos. 3. El aprendizaje servicio. 3.1. Compromiso y utilidad social. 3.2. El ApS y la docencia. 3.3. El ApS, la investigación y la transferencia de conocimiento. 3.4. El ApS y la responsabilidad social. 3.5. El ApS y el emprendimiento social. 4. Proyectos ApS en Comunicación Audiovisual: el caso de la Universidad de Barcelona. 4.1. Criterios para la colaboración. 4.2. Metodología de desarrollo de proyectos ApS. 4.3. Claves del ApS en Comunicación Audiovisual. 4.3.1. Dosificación. 4.3.2. Receptividad. 4.3.3. Beneficio mutuo y colaborativo. 4.3.4. Rigor. 4.3.5. Control. 4.4. Resultados. 4.4.1. Alumnado. 4.4.2. Profesorado. 4.4.3. Institución. 4.4.4. Sociedad. 5. Conclusiones. 5.1. Retos para la universidad. 5.2. Retos sobre el aprendizaje servicio. 5.3. Futuras líneas de trabajo. 6. Referencias bibliográficas.

\title{
1. Introducción
}

La universidad tiene una función social. Es una institución que forma a profesionales capaces de integrarse al mundo laboral, pero también y no menos importante, forma a individuos en competencias y habilidades que les permiten desarrollar valores y virtudes que contribuyen al bienestar social e individual. Para la consecución de estos fines, la llamada psicología positiva ofrece a los docentes una fundamentación científica con que orientar la planificación de su actividad académica. Esta teoría psicológica entiende la conducta humana desde las denominadas fortalezas de carácter, y propone seis categorías universales de fortalezas en torno a las cuales se pueden clasificar virtudes o puntos fuertes del carácter. Dichas fortalezas son: sabiduría, coraje, humanidad, justicia, templanza y trascendencia. A partir de esta clasificación, los individuos — profesores y estudiantespueden identificar los puntos fuertes de su carácter y trabajar a partir de ellos (Peterson y Seligman, 2004).

En este trabajo presentamos una experiencia docente que comparte algunos de los presupuestos de la psicología positiva. Las ciencias sociales y humanas son especialmente adecuadas para pensar en el aprendizaje desde esta corriente psicológica, ya que sus contenidos, competencias y habilidades están en buena medida relacionados con valores. En concreto, abordamos el caso de una práctica formativa del grado de Comunicación Audiovisual que se imparte en la Universidad de Barcelona (UB). Desde 
el curso 2009-2010, este título aspira a formar a profesionales del sector audiovisual en un conjunto de perfiles: director, guionista, productor, realizador, diseñador de producción y posproducción visual y sonora, investigador, especialista en comunicación corporativa y community manager.

En el grado de Comunicación Audiovisual partimos de una concepción de la comunicación no limitada a la mera transmisión de información. Cuestionamos el modelo lineal de la comunicación, que durante décadas ha dominado la investigación en esta área y que también lo ha hecho en el modelo educativo, especialmente en el universitario. Una de las consecuencias derivadas de este enfoque es un excesivo énfasis en la transmisión de contenidos que quizá no presta la atención suficiente a los valores y a la educación emocional asociada. A ese modelo lineal se suele contraponer un modelo de la comunicación como comunidad: commūnicāre, en latín, significa precisamente poner en común, crear comunidad. Y esa es, para algunos autores, la función principal de la comunicación: establecer lazos sociales, crear una cultura compartida (Carey, 1989; Craig, 1999). En este sentido, los valores juegan un papel primordial.

De hecho, creemos que el aprendizaje no puede articularse solo en torno a la transmisión de contenidos y que debe tener en cuenta el papel de las emociones y de los valores en la conducta inteligente. Aprender a través de proyectos de aprendizaje servicio (ApS) permite tener en cuenta las fortalezas de carácter de los estudiantes, la dimensión social de la conducta inteligente, y la función del lenguaje y de la racionalidad en tanto que herramientas que sirven para relacionarnos con los demás. El ApS se enmarca, por tanto, en una corriente innovadora en el ámbito educativo que trasciende a la propia institución educativa, se proyecta a la comunidad y genera con ello un cambio social. Mediante el ApS, la universidad puede ofrecer a los alumnos la oportunidad de intervenir allí donde hay necesidades sociales.

\section{Hipótesis y objetivos}

Este trabajo tiene un doble propósito. Por un lado, pretende caracterizar el ApS desde la perspectiva de la psicología positiva, siempre partiendo de la hipótesis de que se trata de un modelo de aprendizaje capaz de articular la formación emocional y de valores en el ámbito de la Comunicación Audiovisual. Y, por otro lado, describe la experiencia que el Grupo de innovación docente consolidado en Comunicación y Medios Audiovisuales (In-COMAV), formado por un equipo docente multidisciplinar, desarrolla desde 2016 en el grado de Comunicación Audiovisual de la UB con el objetivo de impulsar y de consolidar los proyectos ApS en el grado. En el marco de esta experiencia, a continuación, presenta los criterios de viabilidad establecidos para la aceptación de proyectos de colaboración, los factores críticos de éxito y los resultados que la iniciativa ha tenido sobre el alumnado, el profesorado, la institución y la sociedad. Por último, señala una serie de desafíos y limitaciones que deben superarse para garantizar la viabilidad y la eficacia de los proyectos ApS en la enseñanza superior.

\section{El aprendizaje servicio}

El ApS es una nueva forma de promover el aprendizaje teniendo en cuenta necesidades sociales del entorno que han de ser identificadas por los alumnos, y también por los profesores. Es una metodología con dos facetas que, combinadas, posibilita en el alumnado perfilar un compromiso vital en la medida en que hay una implicación social. Ayuda a adquirir competencias técnicas al mismo tiempo que ayuda al desarrollo de las transversales, en especial las competencias cívicas necesarias para ser buenos profesionales (Hwang, Liu y Salusso, 2019). Y, de este modo, es también una manera de aprender a emprender. 
No en vano, los estudiantes, sin abandonar sus objetivos curriculares, aprenden y crecen académicamente con el espíritu, la motivación y el coraje de las personas emprendedoras que desarrollan sus fortalezas: el ApS, por cuanto nace de la necesidad de encontrar, diseñar e implementar soluciones a problemas reales, los lleva a sentirse capaces de impulsar trasformaciones sociales con que aportar prosperidad y bienestar a la sociedad. Lograr este cometido implica saber identificar oportunidades, superar carencias, construir conocimiento y desarrollar habilidades de forma significativa, optimizando recursos y buscando la calidad y la excelencia. Todo ello son conductas inteligentes y valores educables, propios de un espíritu emprendedor, que han de partir de las fortalezas de carácter de cada estudiante para seguir nutriéndolas, y que son aplicables a su entorno vital y profesional (Sobrado y Fernández, 2010). En definitiva, los estudiantes aprenden mientras adquieren compromisos vitales, satisfacen una necesidad social real, aprenden a emprender y valoran la trascendencia de sus acciones.

El ApS despliega así todo su potencial formativo desde las perspectivas de compromiso y utilidad social, docencia, investigación y transferencia de conocimiento, responsabilidad social y emprendimiento, que asociamos a los objetivos, a los valores y a la función social de la universidad.

\subsection{Compromiso y utilidad social}

Aunque el sistema educativo se ha diseñado en torno al conocimiento explícito, aquel que permite recopilar y archivar información para ser usada en razonamientos posteriores, para autores como Sternberg (1998) la sabiduría reside en el conocimiento tácito, en el saber procesal, en el saber hacer. Este tipo de conocimiento no se adquiere directamente de los demás, y está vinculado con los objetivos y metas que la persona valora. Son las habilidades que se adquieren de la experiencia de vida. La educación debe atender al conocimiento tácito, es decir, debe ayudar a desarrollar habilidades de percepción social y emociones sociales que nos permitan percibir automáticamente lo que es correcto hacer en cada situación. Y el aprendizaje a través de ApS posibilita la adquisición de este tipo de conocimiento.

De acuerdo con Haidt (2006), aquello que solemos llamar pensamiento racional ha evolucionado en los humanos para apoyar conductas automatizadas e intuiciones. La función principal de la racionalidad no es, pues, buscar creencias verdaderas, sino crear interpretaciones que apoyen las creencias más intuitivas, aquellas basadas en las emociones. Por otra parte, según Haidt, son dos los componentes de las condiciones de vida: el amor y el compromiso vital. En nuestra sociedad, el compromiso vital suele llevarse a cabo a través del trabajo, y hay profesiones que permiten desarrollarlo mejor que otras. Son ejemplos de ello la medicina, la comunicación, la educación, las ciencias o las artes, entre otras.

El desarrollo de proyectos ApS es un camino efectivo hacia el compromiso vital, ya que la metodología de trabajo suele incluir objetivos orientados a lograr un impacto positivo en algún ámbito de la sociedad. El ApS es una propuesta educativa que combina procesos de aprendizaje y de servicio a la comunidad en un único proyecto en el que los participantes se forman a la vez que trabajan sobre necesidades reales del entorno con la finalidad de mejorarlo (Mendía, 2012). Es un instrumento educativo con utilidad social, capaz de potenciar el aprendizaje, la participación y el desarrollo de competencias básicas y transversales.

El ApS parte de elementos bien conocidos: el aprendizaje de contenidos, competencias, habilidades y valores, y el servicio voluntario a la comunidad. La innovación, pues, no reside en estos elementos por separado, sino en su estrecha vinculación, materializada en una única actividad educativa bien 
articulada, coherente y de mayor originalidad en la que el propósito pedagógico se funde con la intención solidaria (Puig, Batlle, Bosch y Palos, 2007; Martínez, 2009). La actividad voluntaria, en particular, contribuye de manera efectiva a la felicidad personal, ya que es, según Haidt (2006), uno de los factores externos que la impulsan.

\subsection{El ApS y la docencia}

La labor docente ha experimentado una serie de innovaciones que intentan incorporar a la formación universitaria la dimensión emocional y los valores (Torre y Tejada, 2006). El ApS, orientado hacia esa dimensión, es una innovación que ha llegado a los centros educativos tras otras novedades destacables como la didáctica de los escritos (ensayos) realizados por alumnos, los seminarios especializados y las simulaciones (informáticas y vivenciales). Incide, en concreto, en la convicción de que la docencia no puede limitarse a formar a alumnos encerrados en un aula (Rodríguez y Tiana, 2015). La docencia también requiere abrir la puerta y salir para que el alumnado pueda contribuir en necesidades del entorno y pueda ayudar así a fortalecer el compromiso vital. Al mismo tiempo, trabajar con ApS mejora el compromiso vital del profesorado. Se crea una estructura de trabajo en la que el cuerpo docente, en un mestizaje entre estudiante, profesor y agentes sociales externos, se siente más comprometido con los alumnos, ya que la actividad que estos desempeñan tiene una repercusión social al relacionarse con el entorno como lo harían en un contexto profesional.

Incorporar la metodología ApS en la docencia no solo rompe las paredes del aula, sino que también ayuda a romper los límites típicamente asociados a asignaturas específicas. Con ello, ayuda a superar al mismo tiempo dos barreras, en cierto modo, autoimpuestas por el habitus imperante en la tradición académica universitaria, en el diseño de los planes de estudio y en las formas de evaluación con las que convivimos (Trigueros, Ribera y Torre, 2011). Como resultado, la fórmula del ApS fomenta el aprendizaje transversal y aporta varias ventajas formativas:

- ayuda a formar ciudadanos porque los estudiantes se implican en mejorar alguna situación de su entorno;

- contribuye al crecimiento y fortalecimiento personal y social;

- refuerza el aprendizaje activo y significativo: los estudiantes desarrollan trabajo en equipo y aprenden a identificar necesidades, a conceptualizar, estructurar, desarrollar y ejecutar proyectos, y a evaluar resultados.

- potencia la comunicación (crea comunidad) y el trabajo colaborativo, porque cada equipo de trabajo, en la persecución de su objetivo, puede estar formado por estudiantes de diversas asignaturas y de diversos cursos, e incluso, pluridisciplinariamente, por estudiantes de distintos grados universitarios, todos en interacción con profesores y con agentes sociales externos a la universidad.

Es decir, el ApS aprovecha las fortalezas y el potencial de los alumnos, incrementa su compromiso vital a través del trabajo, refuerza el conocimiento tácito, crea comunidad y, en última instancia, incide en el nivel de felicidad de los estudiantes, de los profesores y de la sociedad. Además, impulsa la innovación docente y la búsqueda de alternativas eficaces con que enriquecer y ampliar el modelo de enseñanza en la universidad para lograr estudiantes sólidamente formados, capaces de integrarse en el mundo laboral con conocimientos, habilidades, y valores y actitudes.

En definitiva, el ApS deja atrás la formación universitaria más tradicional centrada en el profesor y orientada hacia la clase magistral, la adquisición memorística de conocimientos y el trabajo individual del alumno. Llega incluso más allá que las metodologías de aprendizaje activo o de construcción del conocimiento (constructivismo social pragmático) que, para acercarse a la realidad, 
simulan entornos reales de aplicación de conocimientos y de desarrollo de competencias y proponen juegos de rol o estudios de caso o resolución de problemas (Jenaro et al., 2013). El ApS no activa esas estrategias de simulación, sino que lleva a los estudiantes $-\mathrm{y}$ a los profesores - a la realidad, a las necesidades reales que reclaman soluciones viables y de impacto.

De este modo, el ApS se alinea con otras metodologías activas como el aprendizaje basado en retos (Observatorio de Innovación Educativa, 2016), que también mueven al estudiante a involucrarse a investigar y a actuar en un entorno real, a relacionar áreas de conocimiento y a crear entornos de reflexión sobre su aprendizaje. Comparte con estos enfoques el hecho de que el verdadero protagonista es el aprendiz, con sus rasgos de personalidad y fortalezas de carácter; pero también lo es el profesor, que retoma un rol de protagonista en la medida en que pone hincapié en la colaboración con los estudiantes, más que en las calificaciones, así como en su actividad de guía que los apoya en sus decisiones y que los acompaña en la creación de sus estructuras cognitivas y esquemas mentales, necesarios para analizar y comprender la realidad y desarrollar un pensamiento crítico y complejo (Karabenick y Collins-Eaglin, 1997): existen evidencias de que el fomento de este tipo de enseñanza-aprendizaje colaborativo es útil para promover comportamientos prosociales entre estudiantes universitarios (Jenaro et al., 2013). En cualquier caso, el ApS, frente al aprendizaje basado en retos o frente a otras metodologías activas, no descuida nunca su compromiso social y solidario. En el ApS también son protagonistas los agentes sociales.

\subsection{El ApS, la investigación y la transferencia de conocimiento}

El ApS permite, asimismo, abordar la investigación desde la dimensión emocional, partiendo de las fortalezas de carácter de los estudiantes, potenciando el conocimiento tácito y el razonamiento motivado (aquel que apoya nuestras intuiciones). Los proyectos conceptualizados en el marco de esta propuesta desarrollan capacidades investigadoras porque implican un análisis de la sociedad orientado no solo a identificar necesidades sociales, sino también a describirlas y argumentarlas, para luego, además, transferir ese conocimiento a la sociedad con la formulación de una propuesta de ideas canalizada hacia la transformación de algún aspecto de la realidad investigada. El estudiante siente en todo momento que la investigación ha de ser significativa y rigurosa, en la medida en que esas ideas se decantan en un proyecto que necesariamente se conceptualiza, se estructura, se desarrolla, y después se ejecuta y, finalmente, se evalúa.

El ApS constituye, entonces, una labor de investigación científica y de transferencia de conocimiento que, además, aporta al estudiante el manejo de unos procesos y la evaluación de unos resultados que después podrá aplicar en contextos diversos. Investigar implica analizar una parcela de la realidad, identificar problemas, causas y efectos, distinguir la apariencia de la esencia, formular hipótesis y finalmente buscar soluciones. Esa labor investigadora dota a los estudiantes de instrumentos para ser analíticos, críticos y objetivos, y para descubrir vías en la resolución de problemas. Todo ello, dentro de un contexto en el que los valores de compromiso vital, de servicio a la sociedad, de creación de comunidad y de beneficio a la comunidad juegan un papel fundamental. Se trata, por lo tanto, de un tipo investigación caracterizada por una dimensión profesionalizadora y fundamentada en una dimensión emocional. Las fortalezas de carácter descritas por la psicología positiva — sabiduría, coraje, humanidad, justicia, templanza y transcendencia- entran en juego.

Aunque no todos los proyectos de investigación y de transferencia de conocimiento tienen la misma amplitud ni el mismo potencial formativo y de investigación, el ApS se puede aplicar en prácticamente cualquier ámbito académico y social. De hecho, de algunos proyectos ApS han surgido proyectos de investigación destacables (p. ej., el desarrollo de un sistema de rastreo de 
componentes informáticos o la mejora del sistema logístico de un banco de alimentos), y viceversa, de ciertos avances investigadores han surgido proyectos ApS (p. ej., aprovechar tecnología obsoleta de telecomunicación para el desarrollo de poblados de las selvas de Latinoamérica, donde una empresa no invertiría).

Con todo, para que el ApS pueda abordar la dimensión investigadora es esencial que cuente con el apoyo de la institución y de todo el equipo docente (Furco, 2001). Institucionalizar el ApS es un proceso complejo que requiere la coordinación estratégica de varias áreas - la propia misión institucional, la asociación con agentes del entorno, y las cuestiones académicas y estructuralesque deben operar de manera sinérgica para integrar el ApS en el tejido académico de las universidades (Furco, 2008).

\subsection{El ApS y la responsabilidad social}

La responsabilidad social que promueve la filosofía ApS fomenta el compromiso vital de quienes participan en este tipo de iniciativas. El compromiso vital se desarrolla impulsado por el compromiso social, que es el conjunto de acciones que van más allá de nuestras obligaciones legales en relación con la sociedad y con el planeta. Implica la mejora de la calidad de vida de las personas y de cada individuo, y también la mejora del medio ambiente.

En el ámbito empresarial, la responsabilidad social implica generar riqueza, pero también actuar con ética. Este deber no tiene que ser exclusivo del ámbito privado; también afecta al público y, por lo tanto, a la universidad pública, que también ha de ejercerlo porque tiene una responsabilidad enorme e ineludible en su cometido de formar profesionales y ciudadanos.

La responsabilidad social universitaria comenzó en América Latina en la década de los 2000 y se extendió hacia Europa. En España, las primeras grandes concepciones de responsabilidad social las tenemos a partir de 2006, con un criterio que va más allá de la acción social, la extensión universitaria y el voluntariado. Es una reformulación de la universidad (de sus valores, sus iniciativas y sus políticas de gestión), en conjunto, como institución e implica un mayor compromiso con la sociedad y la contribución a un nuevo modelo de desarrollo más equilibrado y sostenible (Universidad Construye País, 2006). Por lo tanto, las acciones que la universidad desempeña han de conducir a algún grado de transformación social y el personal docente debería poder infundir esta filosofía en el alumnado.

La responsabilidad social se puede medir de acuerdo con un conjunto de indicadores: el fomento del buen gobierno y de la transparencia, el desarrollo sostenible, la equidad de género, la inclusión social, el clima laboral y la conciliación, la vida saludable, el consumo responsable y el comercio justo, la economía social y la economía circular, las metodologías activas y participativas, el voluntariado y el ApS (Andres, Barberá y Pallarés, 2017). Estos indicadores aparecen en cuestiones de responsabilidad social a escala europea y también en materia de responsabilidad social universitaria en el ámbito latinoamericano. En este contexto, el ApS aparece como elemento clave, y no solo como metodología docente; es un instrumento imprescindible para incidir en este modelo de innovación y de transformación social. Hacen falta ciudadanos más implicados con la sociedad y más dispuestos a contribuir a su desarrollo y a su cohesión.

Por lo tanto, el ApS no son unas meras «prácticas solidarias». Conlleva una implicación mayor que debe contribuir a transformar, no solo al estudiante, sino también a las instituciones. Por ello, es conveniente que existan proyectos de toda índole, que tanto pueden estar vinculados a la docencia 
como a la investigación. Y que, una vez identificadas las necesidades sociales, impliquen el diseño, la aplicación y la evaluación de las acciones a desarrollar.

Para todo ello es clave establecer vínculos con las entidades del territorio. Si se desea una transformación social, es necesario preguntar a las empresas y a las instituciones culturales, deportivas y sociales del entorno más cercano qué necesidades tienen. Conviene, pues, salir del contexto universitario y escuchar la voz de las entidades de la calle. Al trabajar en red se fomenta la creación de alianzas y se cumple así con el n. ${ }^{\circ} 17$ (meta 17.17) de los Objetivos de desarrollo sostenible (ODS) de las Naciones Unidas (2015): alianzas para lograr objetivos. Igualmente, contribuye a alcanzar el n. 4 (metas 4.3 y 4.4): educación de calidad; e indirectamente puede potenciar y favorecer otros objetivos como el n. ${ }^{\circ} 8$ (meta 8.3): trabajo decente y crecimiento económico; el n. ${ }^{\circ} 9$ (meta 9.5): industria, innovación e infraestructura; y el n. ${ }^{\circ} 11$ (meta 11.4): ciudades y comunidades sostenibles. Además, el ApS colabora directamente en la consecución del objetivo n. $^{\circ} 5$ (meta 5.5), igualdad de género, que en el momento actual se presenta como una punta de lanza para cambios sociales relevantes. Como señala Fernández González (2016), «el convencimiento de que el ApS es una buena estrategia para implicar al estudiantado en los problemas sociales lleva a plantearlo como una herramienta para paliar la discriminación de género».

\subsection{El ApS y el emprendimiento social}

En el contexto europeo de educación las directrices actuales contemplan cinco nuevas cualificaciones. El espíritu emprendedor es una de ellas: tecnologías de la información, lenguas extranjeras, cultura científico-tecnológica, espíritu emprendedor y habilidades sociales. La adquisición de las actitudes emprendedoras debe fomentarse en todos los niveles educativos y, especialmente, en la comunidad universitaria, en todos los ámbitos. Sin duda, combinar el potencial formativo y científico de las universidades mediante el desarrollo de competencias emprendedoras posibilita alcanzar progresos en la mejora y en el avance de la sociedad actual (Sobrado y Fernández, 2010).

Las competencias emprendedoras pueden entenderse en un sentido estrecho como concepto dirigido a la creación de empresas. Y en un sentido amplio, como concepto dirigido al domino de las capacidades y actitudes empresariales, lo que incluye el desarrollo de ciertas cualidades personales y de ciertos puntos fuertes de carácter que conforman el espíritu emprendedor o espíritu de empresa. En este segundo sentido, la Propuesta de recomendación del Parlamento Europeo y del Consejo sobre las competencias clave para el aprendizaje permanente (2005) define este espíritu como:

[...] la habilidad de la persona para transformar las ideas en actos. Está relacionado con la creatividad, la innovación y la asunción de riesgos, así como con la habilidad para planificar proyectos con el fin de alcanzar objetivos. En esta competencia se apoyan todas las personas en la vida cotidiana, en casa y en la sociedad [...], y es el cimiento de otras capacidades y conocimientos más específicos que precisan los empresarios al establecer una actividad social o comercial. 
Desde la universidad, el ApS contribuye a reforzar la creación de una cultura emprendedora entendida en su sentido amplio (Gutiérrez, 2006; Espíritu, González y Alcaraz, 2012). Lo hace integrando las dimensiones de compromiso y utilidad social, docencia, investigación y responsabilidad social que hemos descrito. Y lo hace también edificando comportamientos individuales que, en la perspectiva de la psicología positiva, fomentan el compromiso vital de cada estudiante a través de la asunción de un compromiso social: su comportamiento individual como estudiante tiene que ir alineado con una acción colectiva responsable encaminada a producir una transformación y una mejora en un sector de la sociedad. El emprendimiento social lo consideramos en este sentido.

El ApS universitario no pone, pues, el acento en el desarrollo de las capacidades específicas vinculadas al desarrollo profesional de un empresario, y no persigue con el desarrollo de los proyectos la creación de una empresa o la obtención de beneficios económicos. Al contrario: muchas veces actúa allí donde las empresas no lo hacen porque no consiguen un rendimiento económico.

Con todo, no pierde de vista que tanto la sociedad como el mercado laboral necesitan profesionales con perfil emprendedor que también dibujen un perfil empresarial, adaptables a la enorme evolución de las tecnologías de la información y comunicación, orientados hacia un trabajo cada vez más colaborativo, interdisciplinario y con capacidades de comunicación y de divulgación de su conocimiento experto. Por ello, fomentando el espíritu emprendedor del estudiante en un sentido amplio, el ApS también le ofrece al estudiante los fundamentos para seguir formándose, si así lo siente, en el espíritu emprendedor que lleva a la creación de empresarios que innovan, que asumen riesgos, que combinan recursos, que conciben nuevas formas de hacer las cosas, que transforman su entorno y que persiguen beneficios económicos, siempre desde los principios éticos de servicio social que el ApS contribuye a desarrollar. El espíritu emprendedor del ApS es la base del espíritu empresarial que no abandona, en su pretensión de generar riqueza, la responsabilidad social.

\section{Proyectos ApS en Comunicación Audiovisual: el caso de la Universidad de Barcelona}

Las profesiones relacionadas con la comunicación pueden facilitar el compromiso vital y, por lo tanto, contribuir a la felicidad. En un contexto caracterizado por la evolución tecnológica, la globalización y la orientación hacia un trabajo cada vez más colaborativo e interdisciplinar, el sector audiovisual plantea nuevas exigencias profesionalizadoras. En este sentido, es fundamental que los estudiantes, futuros profesionales, cuenten con las habilidades necesarias para enfrentarse con éxito a los desafíos que presenta el sector audiovisual que, desde nuestro punto de vista, no puede limitarse a la simple elaboración de productos audiovisuales que sean competitivos en el mercado.

De hecho, también son desafíos aprender a constituir equipos de trabajo eficaces y consolidarlos; construir experiencias de liderazgo y de toma de decisiones que mejoren la calidad de vida de nuestras sociedades; y fortalecer rasgos de carácter que impulsen la tenacidad, la templanza, el coraje, la autoconfianza para resolver los problemas que surgirán en el desarrollo de una solución innovadora que se implementará en entornos cada vez más cambiantes. Los estudiantes que participan en un ApS se enfrentan a estos desafíos en un entorno de trabajo que es real, con oportunidades y limitaciones que también existirán en sus nuevos entornos de trabajo tras graduarse.

Los beneficios de los proyectos ApS nos mueven a promover la metodología ApS, a consolidar su implantación en el grado para desarrollar valores y competencias sociales y profesionalizadoras y, por tanto, a considerar todas las propuestas externas que nos llegan desde la necesidad social. 


\subsection{Criterios para la colaboración}

En los últimos años, el equipo docente del grado de Comunicación Audiovisual ha observado un incremento del número de propuestas externas de colaboración. Entidades de variada índole, tanto de la esfera universitaria como de otros ámbitos, solicitan la implicación de alumnos y de profesores del grado de Comunicación Audiovisual para desarrollar un producto audiovisual en el contexto de una campaña de comunicación. Como es bien sabido, los contenidos audiovisuales tienen un gran poder de captación y de persuasión y, motivadas por esta convicción, son muchas las organizaciones que ven en el audiovisual un medio eficaz para transmitir un mensaje.

Sin embargo, no todas las iniciativas tienen el espíritu formativo y de compromiso social que las haga encajar en el modelo educativo del ApS. Para que puedan prosperar en este contexto, las propuestas de colaboración deben contar con ciertas garantías. En particular, deben tener el potencial de aportar al alumnado, al profesorado o al departamento universitario un beneficio curricular que compense la inversión necesaria de tiempo, esfuerzo y recursos. Como parte del aprendizaje, el alumnado ha de tener un claro protagonismo en la concepción de la obra audiovisual y en los procesos de investigación y producción asociados. En consecuencia, la colaboración no puede establecerse en la forma de un encargo cuyo desarrollo se rija por unas directrices de trabajo más propias de una relación comercial. De hecho, es voluntad del equipo docente evitar la competencia desleal con los profesionales del audiovisual, ya que, a nuestro entender, este tipo de iniciativas no debería sustituir el trabajo que en realidad compete a productoras y otras empresas audiovisuales.

Por estas razones, el Consejo de Estudios del citado grado aprobó en mayo de 2018 un conjunto de directrices con el fin de determinar la conveniencia y la viabilidad de implicar alumnado y profesorado del grado en propuestas de colaboración externas. Desde entonces, estas directrices sirven como criterio de valoración (In-COMAV, 2020) y exigen verificar el cumplimiento de un conjunto de condiciones:

- La iniciativa ha de tener un interés pedagógico y académico, y debe permitir el desarrollo de competencias transversales y específicas de la titulación.

- El producto audiovisual resultante debe tener una repercusión social, política, cultural o económica, y ha de contar con el aval de un organismo público, una entidad social o una empresa.

- La propuesta ha de poder materializarse en un proyecto de mejora e innovación docente, de aprendizaje servicio, de transferencia de conocimiento o de investigación, en el cual debe estar implicado profesorado del área de Comunicación Audiovisual del departamento que gestiona la titulación.

- La propuesta ha de poder desarrollarse de forma natural en el marco de una asignatura, desde el punto de vista tanto académico como cronológico (un semestre académico o, en casos excepcionales, un curso académico) y debe poder contar con la figura de un tutor.

- La colaboración no puede quedar limitada a la realización del producto audiovisual, sino que debe incluir también una tarea de investigación y conceptualización de la obra a realizar.

- El producto no puede tener como finalidad principal un beneficio comercial o un provecho exclusivo para la entidad promotora; no puede ser, p. ej., un anuncio publicitario o un vídeo promocional. En caso afirmativo, si la propuesta proviene de la propia universidad, la fórmula adecuada es la convocatoria de becas de colaboración.

- La propuesta debe aportar unos recursos, que tanto pueden ser una dotación económica (beca de colaboración, dietas, alquiler de material, etc.) como algún beneficio, en términos de proyección profesional o institucional, para el alumnado del grado de Comunicación Audiovisual y para el departamento que gestiona la titulación. 
- La actividad desarrollada por los estudiantes no puede incurrir en competencia desleal con el sector profesional. El destinatario del servicio debe aceptar que los estudiantes desarrollan una actividad de aprendizaje en el marco de su formación universitaria y, por lo tanto, podrían cometer errores y el producto final podría no ser satisfactorio. Aunque se velará por la calidad del servicio, en ningún caso el proyecto sustituirá la prestación de un servicio profesional.

\subsection{Metodología de desarrollo de proyectos ApS}

La puesta en marcha de proyectos ApS en el marco del mencionado grado de Comunicación Audiovisual suele venir determinada por las propuestas de colaboración que se reciben de agentes externos. La demanda es tan elevada que impide establecer una labor sistemática de diagnóstico del entorno y de sus necesidades. En su lugar, pues, se prefiere basar la elección de proyectos en la evaluación minuciosa de las propuestas recibidas.

Para ello, con la persona que representa a la entidad promotora se concierta una entrevista para determinar, de acuerdo con las directrices descritas antes, si la propuesta reúne las condiciones necesarias y si es compatible con el modelo educativo del ApS. Esta entrevista de referencia ayuda a saber más sobre la institución y sobre el compromiso social del potencial proyecto, y en ocasiones permite ajustar los objetivos o el alcance del proyecto para consolidar su viabilidad.

En los casos en que se considera necesario (p. ej., si hay dudas sobre tal viabilidad), una comisión formada por el jefe de estudios, el jefe de departamento y al menos un profesor del área de la Comunicación Audiovisual pueden emitir una valoración definitiva sobre la conveniencia de aceptar o rechazar una propuesta.

Si la propuesta está alineada con las mencionadas directrices, se añade a la cartera de propuestas y se presentan todas ellas a los estudiantes de las asignaturas Proyectos II y TFG, las que mejor pueden acoger este tipo de proyectos. Cabe tener en cuenta que los estudiantes reciben las propuestas hasta tres meses antes de que empiecen a desarrollarlas. Tienen tiempo suficiente de elegir una que les atraiga. Saben, además, que su elección constituirá la evidencia de evaluación de una de sus asignaturas y que, antes de conocer al interlocutor externo de la entidad promotora, se les pedirá un compromiso personal y de equipo, una implicación máxima y el coraje de aventurarse en una metodología activa que rompe con esquemas tradicionales de enseñanza-aprendizaje.

Si todo ello se cumple y se cuenta con un profesor dispuesto a tutorizarlo, se cita a los alumnos y al tutor con el representante de la entidad promotora para una primera toma de contacto, el proyecto recibe la aprobación definitiva, se firma un convenio de colaboración que convierte la iniciativa en un proyecto ApS y se exige que el producto final refleje en los títulos de crédito la participación del grado de Comunicación Audiovisual.

La entidad promotora interviene en el desarrollo del proyecto y tiene voz para decidir sobre la adecuación del producto audiovisual final que motiva el proyecto. También valora el resultado de la obra audiovisual. Cuando da su aprobación, la obra audiovisual sale propiamente del entorno universitario y adquiere entonces razón de ser por su utilidad en el entorno social que la reclama. Hasta el momento ningún vídeo ha sido valorado como inadecuado o erróneo. Solo en alguna ocasión se han pedido ajustes menores - algún cambio de diseño o de grafismo-, motivados por un cambio en el contexto social (por ejemplo, el confinamiento en tiempos de la covid-19). 
Por otro lado, también es habitual que la entidad promotora organice algún acto para presentar la obra, por ejemplo, en centros cívicos, en algún congreso o en el marco de una fecha significativa (un aniversario) para la entidad. La entidad se encarga luego de difundir esa obra por canales tan diversos como su sitio web o la sala de espera de un hospital público.

Concretamente, uno de los hospitales con los que se ha venido colaborando ha fidelizado su participación en proyectos ApS por la buena acogida que las obras audiovisuales resultantes están teniendo entre los pacientes. De hecho, en la actualidad se está planificando una investigación conjunta, en la que también participan estudiantes, para medir el impacto de los mensajes que divulgan las obras audiovisuales en el grado del cumplimiento de los tratamientos indicados a pacientes que atienden su turno en las salas de espera en las que se proyectan los vídeos. Incluso un nuevo hospital se ha interesado por intervenir en proyectos ApS del grado de Comunicación Audiovisual.

También en la facultad, paralelamente, se evalúan los proyectos. Los estudiantes han de defender la viabilidad y la pertinencia de su trabajo ante un tribunal de profesores que valora su capacidad de comunicación oral persuasiva. Y lo que es más: tras recibir la calificación final, el siguiente paso es que los estudiantes difundan sus proyectos y productos audiovisuales en jornadas y congresos nacionales e internacionales en los que se trata sobre innovación docente universitaria o sobre divulgación del conocimiento experto o científico. De este modo, también desarrollan habilidades de comunicación persuasiva no solo en entornos académicos, sino también en entornos científicos y profesionales.

Esto implica que los proyectos ApS se prolongan forzosamente más allá de un curso académico, que estudiantes y profesores buscan fuentes de financiación para asistir a esos congresos (se ha conseguido que una empresa privada financie la participación de cuatro estudiantes en un congreso de farmacología en Estados Unidos), y que los estudiantes siguen aprendiendo sin que a cambio reciban una nota en su expediente académico: su recompensa es la experiencia y el aprendizaje adquiridos. También los estudiantes que han participado en proyectos ApS se ofrecen para tutorizar a estudiantes que se inician en ellos.

\subsection{Claves del ApS en Comunicación Audiovisual}

Tres años de experiencia en la planificación e implementación de proyectos ApS nos permiten identificar una serie de factores críticos de éxito.

\subsubsection{Dosificación}

Es conveniente que la iniciativa ApS se incorpore de manera gradual y dosificada para que los resultados, y no solo los procesos, sean los adecuados. De este modo se puede garantizar la continuidad y la sostenibilidad de la innovación. Al fin y al cabo, el ApS forma parte de un discurso y de una práctica de innovación en educación que requiere de una infraestructura.

Se valora más un proyecto acabado que diez empezados sin visos de finalizar. Es peligroso querer dar salida a absolutamente todo, especialmente si el grado apenas ha comenzado a aplicar la fórmula del ApS, porque en esa fase incipiente, aunque pueda haber estudiantes dispuestos, carece de una infraestructura adecuada. Es mejor, pues, comenzar implicándose en un gran proyecto, quizá con muchos estudiantes, que iniciarse en varios proyectos pequeños, ya que el trabajo de gestión se multiplica en cada proyecto nuevo. 


\subsubsection{Receptividad}

Dado que desde la universidad es difícil conocer todas las necesidades del entorno, a fin de detectarlas conviene hacer un ejercicio de humildad y estimular la sensibilidad social. La universidad debe saber escuchar, debe entender cuál es la necesidad particular de cada contexto y debe tener claro que su papel no es imponer soluciones, sino encontrar la manera de ayudar a resolver los problemas.

La universidad no debe trabajar para los agentes del entorno, sino que ha de trabajar con ellos, de igual a igual, ya sea otra facultad, un barrio desfavorecido, un departamento médico o cualquier otra institución. En este sentido, el grado de Comunicación Audiovisual se presenta como un espacio de comunicación que propone creatividades a medida.

\subsubsection{Beneficio mutuo y colaborativo}

Más allá del impacto positivo que el ApS tiene en la sociedad, en este contexto de colaboración, todas las partes implicadas directamente - alumnado, profesorado e institución - han de salir ganando, y el beneficio que obtendrá cada parte debe quedar definido con precisión.

La actividad que ejerce el alumnado, de servicio a la comunidad, debe tener un claro componente formativo. Los estudiantes no pueden acabar convertidos en mano de obra gratuita. De hecho, se rechaza un gran número de propuestas procedentes de instituciones del entorno porque no son proyectos con un componente formativo, sino con el único objetivo de beneficiarse de un trabajo sin honorarios. Asimismo, el producto puede ser objeto de difusión en diferentes medios, así como una entrada curricular, tal y como muestra la filosofía del proyecto. Se ha observado cómo los estudiantes se implican emocionalmente en los proyectos ApS y, en ocasiones, al terminarlos, continúan un trabajo de voluntariado (Einfeld y Collins, 2008).

El profesorado, por su parte, sale del aula y tutoriza proyectos con un «cliente», por lo que debe adaptarse a las necesidades de este y debe guiar el trabajo hacia el objetivo adecuado o pactado. En un grado como el de Comunicación Audiovisual de la UB, donde el $80 \%$ del profesorado es asociado, significa un acompañamiento al estudiante al propio lugar de trabajo. El tutor conoce el camino, y el estudiante lo recorre con la seguridad de que, si surge algún contratiempo, no será irreversible. Y ambos, tutores y estudiantes, deben permitirse la experimentación y la creación, en la medida en que el calendario de trabajo lo permita. No se trata de resolver un problema, sino de concebir un producto desde un contexto social determinado, para incidir en él y mejorarlo.

La institución, como hemos visto, defiende, promueve y acepta proyectos ApS, por lo que la notoriedad que adquiere al incorporar este campo del aprendizaje es amplia, y abarca muchos y muy variados valores: la innovación, la enseñanza transversal, la integración en la sociedad y la reciprocidad. En la filosofía $\mathrm{ApS}$, la universidad encuentra una vía para desarrollarse como institución comprometida con el entorno y para hacer visibles en las misiones de la universidad las acciones desarrolladas por el profesorado. Así como hemos mencionado las fortalezas de carácter de los individuos, cabría también hablar de las fortalezas de las instituciones, en general, y de la universidad, en particular.

El proyecto, pues, se convierte en una situación beneficiosa para todas las partes involucradas de forma directa: además del entorno, salen ganando el alumnado, el profesorado y la institución. 


\subsubsection{Rigor}

Si bien se debe confiar en los alumnos, puesto que suelen poner de su parte para afrontar los retos y superar los problemas, esa confianza debe ir acompañada de un rigor. El aprendizaje debe estar relacionado con las materias del grado y debe tener unos objetivos definidos, lo cual ha de quedar explicitado y ha de contar con mecanismos adecuados de evaluación. Los trabajos de ApS, en la medida en que se inscriben dentro de una asignatura del grado como puede ser la asignatura de Proyectos II (asignatura obligatoria de tercer curso), o el TFG (asignatura obligatoria de cuarto curso), siguen las pautas de evaluación establecidas en los planes docentes correspondientes.

\subsubsection{Control}

Desde el grado de Comunicación Audiovisual se ha intentado asumir todos los proyectos que aportasen el beneficio mutuo mencionado antes (3.2.3). Sin embargo, conscientes de que la fórmula del ApS se encuentra en una fase incipiente - tres cursos lectivos - y carentes de infraestructura, observamos cómo el trabajo de gestión se multiplica con cada nuevo proyecto. Así, se intenta que sean los propios estudiantes quienes gestionen las expectativas de sus «clientes», con el asesoramiento de sus tutores y apoyados en sus compañeros de curso en caso de necesitar ayuda adicional en diferentes roles.

\subsection{Resultados}

En el período de noviembre de 2016 a noviembre de 2019, y en el marco de la metodología ApS, en el grado de Comunicación Audiovisual de la UB se han abordado siete proyectos audiovisuales. Estos proyectos ApS han tenido, cada uno, un objetivo particular:

- la divulgación del tratamiento de las úlceras neuropáticas en el pie diabético;

- una campaña de sensibilización pública contra la automedicación, con énfasis en el consumo responsable de antibióticos;

- la divulgación del tratamiento del pie de atleta (dermatofitosis);

- la difusión de la lengua y la cultura catalanas entre estudiantes Erasmus;

— el lenguaje de sordomudos en catalán para los estudiantes de la universidad;

- la ruptura del estereotipo de la profesión bibliotecaria;

— dos proyectos relativos a la recuperación de la memoria histórica de un barrio desfavorecido.

En pleno curso 2019-2020, iniciando el tercer año de incorporación de ApS en el grado, los proyectos en fase de desarrollo o preproducción son:

- un tercer proyecto de recuperación de la memoria histórica del mismo barrio desfavorecido;

- dos proyectos más, relativos al consumo responsable de medicamentos;

— la divulgación de las aportaciones de una institución marítima que cumple 250 años.

Se observa, por tanto, una continuidad con proyectos realizados anteriormente, lo cual supone un indicador positivo respecto al éxito de este tipo de trabajos entre el alumnado.

También es una señal positiva que hayan sido los propios estudiantes quienes hayan presentado estos proyectos en doce congresos nacionales e internacionales, a los que han asistido con los profesores tutores o, en alguna ocasión, sin estos profesores.

\subsubsection{Alumnado}

Los alumnos que han participado en estas iniciativas han ganado en ilusión y motivación. Son conscientes de que los proyectos que desarrollan tienen una utilidad social y se acaban utilizando en 
campañas de comunicación. Además, perciben el aprendizaje de un modo aplicado, muy práctico; les resulta más ameno y motivador, y ese aprendizaje en la sociedad hace que se puedan realizar más como personas.

Con la dimensión de servicio, los alumnos mejoran sus competencias sociales y, con la dimensión de aprendizaje, desarrollan competencias profesionales. La mayoría de estos efectos positivos pueden categorizarse en alguna de las seis fortalezas mencionadas al inicio. Se aprecia, p. ej., un incremento en el amor por el aprendizaje (sabiduría), liderazgo, ciudadanía (justicia), humor (trascendencia).

Dado que los alumnos eligen los proyectos que más se ajustan a sus perfiles y motivaciones, como indicamos anteriormente, también suelen mostrarse activos en su desempeño: han de identificar necesidades del entorno, aprenden a analizar el contexto, a sintetizar situaciones complejas, a formular objetivos y a diseñar planes de acción. Del mismo modo, aprenden a evaluar: durante todo el proceso reflexionan sobre lo que hacen para mejorar, de manera que, al implicarse en proyectos reales, aprenden a aprender y también aprenden a transferir el conocimiento que generan a otros ámbitos del saber.

Nos encontramos, además, ante un mercado laboral que necesita profesionales con un talante emprendedor (AQU Catalunya, 2018), que es justamente el perfil que mejor define la naturaleza del estudiante que participa en los proyectos ApS. De hecho, el espíritu de un emprendedor se define como una forma de ser, de pensar y de hacer que estimula transformaciones sociales con el fin de mejorar la calidad de vida de los individuos y de aportar prosperidad y bienestar a la sociedad. Su filosofía es la optimización de recursos y la búsqueda de la excelencia.

Con estos propósitos, se ha logrado aumentar el esfuerzo de los estudiantes para lograr la excelencia en la persuasión oral (pitches y presentaciones en congresos), digital (medios sociales) y audiovisual (obras audiovisuales y sus correspondientes teasers y trailers). Y esta excelencia no solo se ha reflejado en el producto de su trabajo, sino también en su satisfacción. Muchos de los estudiantes que en tercer curso se incorporan a un ApS, a través de la asignatura de Proyectos, en cuarto curso esperan poder repetir la experiencia en el Trabajo de Fin de Grado.

Como beneficio adicional, fruto de la motivación que genera la participación en este tipo de iniciativas, los estudiantes involucrados en ellas tienden a obtener calificaciones sensiblemente mejores (véase Tabla 1).

Tabla 1. Calificaciones obtenidas en proyectos ApS y en proyectos no ApS

\begin{tabular}{lcccc}
\hline \multicolumn{1}{l}{ Asignatura - curso académico } & $\begin{array}{c}\text { Proyectos } \\
\text { ApS }\end{array}$ & $\begin{array}{c}\text { Nota media } \\
\text { proyectos ApS }\end{array}$ & $\begin{array}{c}\text { Nota media } \\
\text { proyectos no ApS }\end{array}$ \\
\hline Proyectos II & $2016-17$ & 2 & 9,10 & 7,56 \\
Proyectos II & $2017-18$ & 1 & 8,04 & 6,83 \\
Trabajo de Fin de Grado & $2017-18$ & 2 & 8,40 & 7,48 \\
Proyectos II & $2018-19$ & 1 & 7,31 & 7,35 \\
Trabajo de Fin de Grado & $2018-19$ & 2 & 7,37 & 7,65 \\
De la Idea a la Pantalla & $2019-20$ & 1 & 9,00 & 7,19 \\
\hline & & $\mathbf{9}$ & $\mathbf{8 , 2 0}$ & $\mathbf{7 , 3 4}$ \\
\hline
\end{tabular}

Fuente: Franganillo et al. (2020) 
Los profesores aprecian la satisfacción de los estudiantes por el trabajo realizado. Es de destacar la emoción e implicación con que presentan sus trabajos en las defensas orales: actúan en equipo, como un engranaje bien perfilado, apoyándose mutuamente.

\subsubsection{Profesorado}

Se ha conseguido también intensificar la reflexión didáctica de los docentes sobre el trabajo interdisciplinario y sobre las competencias profesionalizadoras de los estudiantes. En definitiva, se han obtenido mejoras en los procesos de enseñanza-aprendizaje de la titulación.

El trabajo en equipo del profesorado, en comunicación permanente entre ellos, ha sido constante a lo largo de los tres últimos años. Como resultado, ha proporcionado el marco apropiado de cómo y cuándo se dan las circunstancias correctas para incorporar un ApS en las diferentes asignaturas, y cómo tutorizarlos de la forma más adecuada: para el proyecto, para el equipo de trabajo y para la institución externa. En definitiva, se han conseguido mejoras en los procesos de enseñanzaaprendizaje de la titulación.

\subsubsection{Institución}

Las universidades adquieren proyección institucional con este tipo de colaboraciones, ya que ayudan a incorporar a su marca su implicación social y subrayan la estrecha relación entre conocimiento y bienestar social. Así, el tipo de actividad en el que se implican los estudiantes a través de los ApS no solo les beneficia a ellos, sino también a la propia institución, que logra acercarse a las realidades sociales e incidir en ellas. De hecho, las universidades son hoy muy conscientes del valor que tienen las competencias sociales y buscan activamente articular una manera de reconocer esta dimensión en la formación universitaria (Arroyo, 2019).

Es fundamental subrayar que, para conseguir este objetivo, la universidad debe intensificar el filtraje de propuestas para evitar proyectos depredadores sin un verdadero valor para alumnos, profesores o para la facultad. Como institución, debemos, pues, reforzar el rigor en la selección. En nuestro grado de Comunicación Audiovisual de la Universidad de Barcelona, el filtraje es exhaustivo. De hecho, hemos ampliado y priorizado la segunda de las directrices como criterio de selección de propuestas: «Tendrán preferencia las propuestas dirigidas de manera directa o indirecta a colectivos en situación de vulnerabilidad social o en riesgo de formar parte de estos colectivos». Esto último, además, nos alinea mejor con los ODS, que, si bien abordan la igualdad, la prosperidad y la protección del planeta, apelan a la ayuda de los colectivos desfavorecidos.

\subsubsection{Sociedad}

Es obvio, por todo lo dicho, que el otro gran beneficiario de los trabajos ApS es la sociedad. La universidad forma a ciudadanos que no solo adquieren conocimientos, sino que además desarrollan competencias y habilidades sociales, morales, éticas, de compromiso social, atendiendo al gran capital humano que suponen los valores y las virtudes. De este modo, la universidad devuelve a la sociedad lo que esta espera de ella: formar a individuos que contribuyan al bienestar colectivo.

\section{Conclusiones}

La universidad debe desarrollar la capacidad de establecer relaciones con los socios de la comunidad, y debe contribuir a concebir y diseñar los proyectos de colaboración como una experiencia capaz de 
aportar un aprendizaje competencial y vivencial. El énfasis que la psicología positiva pone en la inteligencia emocional, en las fortalezas de carácter, en el conocimiento tácito y en las variables que forman parte de la felicidad o el bienestar es de gran utilidad para repensar la docencia, los procesos de aprendizaje y el papel de la universidad en la sociedad. De hecho, la Universidad de Barcelona (2019) se adhiere a la Agenda 2030, sobre igualdad y acción social, y los proyectos ApS parecen estar alineados con los objetivos de esta agenda.

En diversas reuniones, el rectorado de la UB ha valorado positivamente la intensa actividad relacionada con el ApS. De hecho, la UB es la universidad catalana que más proyectos ApS desarrolla, y es una tendencia al alza. El hecho de que una facultad tenga visión social y colabore con entidades le aporta visibilidad y prestigio. Y el grado de Comunicación Audiovisual es, dentro de la UB, una de las enseñanzas líderes en iniciativas ApS y orientación social. La actividad desarrollada en el marco de la metodología ApS aparece reconocida en el suplemento europeo y redondea el currículum de los alumnos.

Al fin y al cabo, la persona egresada en Comunicación Audiovisual ha de ser un profesional polivalente, proactivo, con iniciativa y capacidad analítica, de resolución de problemas y de toma de decisiones, con capacidad de liderazgo y de trabajo en equipo, y competente en la comunicación persuasiva. Coincide con esta visión el reciente informe de AQU Catalunya (2019), según el cual las competencias transversales más apreciadas por los empleadores son precisamente la formación práctica, la capacidad de resolución de problemas y la habilidad en la toma de decisiones. Asimismo, las competencias específicas más valoradas son la capacidad de comunicar de manera efectiva, la habilidad para idear, planificar y ejecutar proyectos comunicativos, y la capacidad de exponer de forma razonada ideas y argumentos.

En el marco de las colaboraciones ApS, los estudiantes revierten a la sociedad su conocimiento, presentan su proyecto fuera de las aulas, en el contexto en el que lo han desarrollado, y entienden que su proyecto ha hecho una contribución valiosa a un ámbito de la sociedad. La experiencia positiva acumulada durante estos tres años hace que los proyectos ApS se perciban entre el alumnado como experiencias atractivas y significativas.

\subsection{Retos para la universidad}

Otro aspecto que nuestra experiencia con $\mathrm{ApS}$ ha puesto de manifiesto es que la universidad necesita flexibilizar el tipo de figura contractual entre el profesorado, especialmente en aquellas disciplinas vinculadas con perfiles profesionales, tal como ocurre con los estudios de Comunicación Audiovisual.

Es necesario incorporar al cuerpo docente profesionales con experiencia, a pesar de que la carrera académica está pensada para quienes se dedican solo a la universidad. En la universidad española y catalana, la figura contractual concebida para incorporar a profesionales en el profesorado es la figura del profesor asociado, que por contrato tiene una dedicación muy limitada. La docencia a través de proyectos ApS se realiza a través de la tutorización que, como modelo de enseñanza, requiere de dedicación horaria y de atención personalizada, especialmente cuando «el cliente», la institución externa, es exigente. Igualmente, precisa capacidad de decisión profesional, en ocasiones ajena a normativas relacionadas con planes docentes. El impulso de este tipo de modelos de enseñanza dentro de la universidad debería ir acompañado de figuras contractuales distintas que no se centren solo en el valor de la carrera académica, puesto que para el profesional transformado en docente-tutor, la carrera académica suele carecer de valor. 
La relación entre universidad e industria, por su parte, tiene grandes escollos que salvar. La inflexibilidad contractual del profesorado es uno de ellos, pero también lo es la rigidez de los planes de estudios y de los planes docentes. A ello contribuye negativamente el marco para la verificación, el seguimiento, la modificación y la acreditación de los títulos oficiales (VSMA). Aunque se trata de un marco de referencia necesario para garantizar la calidad de la enseñanza, está concebido con una rigidez que limita la capacidad de reacción e impide adecuar los planes de estudios con la rapidez y la flexibilidad suficientes a los nuevos escenarios de la comunicación y a los nuevos perfiles profesionales, que cambian constantemente.

Por lo tanto, la universidad, para ser verdaderamente moderna, debe flexibilizar los procesos de contratación de profesorado y también los procesos académicos. La modernidad no la determinan únicamente las metodologías docentes, sino también la organización académica. Actualmente hay demasiada presión sobre el profesorado y muchas exigencias burocráticas para mostrar que se está modernizando la docencia universitaria, pero en el fondo la actividad docente sigue condicionada por un modelo de universidad excesivamente rígido.

\subsection{Retos sobre el aprendizaje servicio}

La democratización de la universidad no consiste solo en la aceptación de cualquier estudiante con la preparación adecuada, sino también en la capacidad de adaptarse a su entorno, es decir, la capacidad de reconocer que en el firmamento que la engloba se puede incluir un espacio de aprendizaje. Por su parte, la universidad está ampliamente reconocida como el centro del conocimiento, de la elaboración de hipótesis y de la demostración de teorías, un espacio en el que puede encontrar procesos, soluciones y aportaciones a sus causas. Es indudable que, para ambos ámbitos — la universidad y la sociedad-, el ApS es una metodología especialmente apropiada para la educación en valores, que es, de hecho, una de las tareas de la educación reglada.

La Comunicación Audiovisual tiene un lenguaje propio, con códigos conocidamente asentados en la conciencia colectiva - el tipo de planos, el ritmo narrativo, la calidez de la imagen, etc.- -, mediante los que transmite cultura, ideología, identidad y referentes. Al aplicar la Comunicación Audiovisual al ApS se popularizan fácilmente los mensajes y se transforma su difusión, puesto que no se transmiten por los canales de distribución convencionales. Al mismo tiempo, se adaptan las necesidades a las demandas $\mathrm{y}$, al abordar casos reales con clientes reales, se profesionaliza el trabajo universitario.

Las dinámicas de trabajo de los estudiantes se adaptan a los procesos profesionales, que se inician con la reunión con el cliente y acaban con la presentación del trabajo realizado, e incluso aceptando las posibles peticiones de cambios. Sorprendentemente, los proyectos ApS proponen tareas muy activas y motivadoras, aceptadas por estudiantes eficientes y solícitos. En tres años, con una sola excepción desalentadora, resultante de la actitud, más que de la aptitud, del equipo de trabajo, los resultados suelen reflejar rendimientos académicos de alto nivel y suponen una clara ventaja en la incorporación al mundo profesional. En este sentido, cabe destacar que la mayoría de los alumnos implicados en proyectos ApS han participado, y continúan haciéndolo, en congresos nacionales e internaciones de carácter científico o de innovación docente relacionados con sus proyectos; y que algunos de ellos han conseguido contratos de trabajo o, en un caso, incluso han atraído inversores para su primera start-up. Todo ello, gracias a la experiencia adquirida en dichos proyectos. 
Los grados de alto componente creativo y experimental, como Comunicación Audiovisual, pueden inspirar, quizá, prejuicios de poco rigor y mucha bohemia y glamour. Es decir, mucha estética y poco estudio. Pero nada más lejos de la realidad. En este contexto, la figura del ApS instaura una actividad de contenido muy formal en varios planos de máxima relevancia para el crecimiento de estudiante, como persona y como profesional:

a) Por el trato al cliente, al que no se conoce previamente. Al incorporar modales y formalidades poco habituales, hay que llegar a comprender la situación y las necesidades del interlocutor para poder elaborar propuestas y soluciones a su alcance. La escucha debe ser activa, y la comprensión, máxima.

b) Por los procesos de trabajo para conseguir realizar el encargo, que deben ser profesionales en todos los sentidos. El método de trabajo que se enseña en el grado es el que funciona en el sector profesional, y los estudiantes lo adoptan para la consecución de sus objetivos.

c) Por las relaciones entre los componentes del equipo. El mundo audiovisual, profundamente jerarquizado, se refleja en las tareas distribuidas en el equipo. Si bien el entorno académico permite flexibilidad en las responsabilidades, el objetivo del ApS determina unos flujos de «poder»y de «poder de decisión» dentro del propio equipo, cuyas ramificaciones pueden llegar a ser de lo más variado en el desempeño de esos roles.

d) Por la relación de los estudiantes con el tutor de cada proyecto. La confianza depositada en los estudiantes crea un nexo: la comunicación mejora, y el lenguaje entre jóvenes y adultos se aproxima. La tutorización es una guía/sugerencia para el trabajo, no el trabajo en sí mismo. Los tutores reciben un estímulo inspirador: la obra audiovisual.

Esta reflexión final, en definitiva, viene a constatar que el ApS, incorporado al grado de Comunicación Audiovisual de la UB, es una herramienta altamente profesionalizadora que permite acercar al estudiante a su futuro, tanto en el plano personal como en el profesional. La combinación de trabajo académico y práctico con proyectos sociales ha repercutido de forma positiva en el proceso de aprendizaje y en los resultados creativos. Es razonable esperar, pues, que el desarrollo pedagógico de los grados con un notable componente práctico incorpore cada vez más la metodología ApS, fundamentada en los principios de la psicología positiva, como modelo de innovación docente y como instrumento profesionalizador.

\subsection{Futuras líneas de trabajo}

Una línea de trabajo inmediata consistirá en aplicar a los estudiantes del grado de Comunicación Audiovisual de la Universidad de Barcelona el cuestionario VIA Adult Survey (disponible en https://viacharacter.org). Peterson y Seligman empezaron a estudiar las fortalezas en 2001 a partir de este cuestionario en línea, que actualmente recoge hasta 24 fortalezas, y cada una de ellas se valora mediante 10 ítems (Gustems y Sánchez, 2015).

A partir de los resultados obtenidos, podríamos detectar con mayor exhaustividad aquellas fortalezas de carácter (virtudes, valores) que caracterizan a los estudiantes de Comunicación Audiovisual. Con ello, podríamos mejorar propuestas de aprendizaje a través del ApS, y tener una mejor comprensión del perfil de nuestros estudiantes en cuanto a competencias sociales se refiere.

\section{Referencias bibliográficas}

Andrés Martínez, A., Barberá Forcadell, S. \& Pallarés Domínguez, D. (2017). Nuevas perspectivas en la gestión de la responsabilidad social en las universidades. Castelló de la Plana: Publicacions de la Universitat Jaume I. http://guninetwork.org/files/9788416546794.pdf 
AQU Catalunya (2019). La opinión del colectivo empleador sobre la formación de las personas tituladas en Comunicación. Barcelona: AQU Catalunya. https://www.aqu.cat/doc/doc_19921032_1.pdf

Arroyo, J. (2019, 14 de octubre). Un currículum más allá del aula. El País. https://elpais.com/sociedad/2019/10/13/actualidad/1570981445_198065.html

Boix, R. \& Lazzeretti, L. (2012). Las industrias creativas en España: una panorámica. Investigaciones regionales, 22 , 181-206. http://aecr.org/images/ImatgesArticles/2012/5/09_BOIX.pdf

Boix, R., Lazzeretti, L., Capone, F., Propris, L. \& Sánchez, D. (2010). The geography of creative industries in Europe: Comparing France, Great Britain, Italy and Spain. European Urban and Regional Studies, 23(4). https://doi.org/10.4324/9780203112571

Carey, J. (1989). A cultural approach to communication. En D. McQuail (ed.). McQuail's Reader in Mass Communication Theory. Londres: Sage Publications, 37-45.

Comisión Europea (2010). Libro verde sobre el potencial de las industrias culturales y creativas. Bruselas: Dirección General de Educación y Cultura. http://eurlex.europa.eu/legalcontent/ES/TXT/?uri=URISERV:cu0006

Comisión Europea (2019). Eurostat: culture. https://ec.europa.eu/eurostat/web/culture/data/database

Comisión Europea (2019). Business demography by size class (from 2004 onwards, NACE Rev. 2, bd_9bd_sz_cl_r2). https://ec.europa.eu/eurostat/data/database?node_code=bd_9bd_sz_cl_r2

Craig, R. (1999). Communication theory as a field. Communication Theory, 9, 119-161.

Einfeld, A. \& Collins, D. (2008). The Relationships between service-learning, social justice, multicultural competence, and civic engagement. Journal of College Student Development, 49, 95-109. http://doi.org/10.1353/csd.2008.0017

España. Ministerio de Educación, Cultura y Deporte (2016). Cuenta satélite de la cultura en España: avance de resultados, 2010-2014. Madrid: Ministerio de Educación, Cultura y Deporte. http://educacionyfp.gob.es/dam/jcr:fb5482cc-f82f-48f2-ab6f-e2411ebd7f38/cuenta-satelite-de-lacultura-en-espana-2010-2014.pdf

Espíritu, R., González, R.F. \& Alcaraz, E. (2012). Desarrollo de competencias emprendedoras: un análisis explicativo con estudiantes universitarios. Cuadernos de estudios empresariales, 22, 2953.

Eurostat (2016). Government expenditure on recreation, culture and religión. Eurostat: statistics explained. https://ec.europa.eu/eurostat/statisticsexplained/index.php/Government_expenditure_on_recreation,_culture_and_religion

Franganillo, F., Sánchez, L., García, M.A. \& Marquès, A. (2020): Fostering social change through short films: how to train future media professionals in an interdisciplinary and applied way. 
Journal of Higher Education Theory and Practice, 20(12), 106-114. https://doi.org/10.33423/jhetp.v20i12.3782

Furco, A. (2001). Advancing service-learning at research universities. New Directions for Higher Education, 114, p. 67-78. https://cmapspublic3.ihmc.us/rid=1K3GTLWN5-L0TF8PY4X/Furco_A-ServiceLearnResearchUniv.pdf

Furco, A. (2008). Institutionalising service-learning in higher education. En L. McIlrath \& I.M. Labhrainn (eds.). Higher Education and Civic Engagement: International Perspectives. Farnham: Ashgate Publishing, 65-82.

Gustems, J. \& Sánchez, L. (2016). Aportaciones de la psicología positiva aplicadas a la formación del profesorado. Estudios sobre Educación, 29, 9-28. https://doi.org/10.15581/004.29.9-28

Gutiérrez, F. (2006). Desarrollo local-endógeno y el papel de las universidades en la formación de cultura emprendedora e innovadora en territorios socio-deprimidos, Laurus, 12(22), 139-152.

Haidt, J. (2006). La hipótesis de la felicidad. Barcelona: Gedisa.

Hwang, C., Liu, H. \& Salusso, C.J. (2019). Social responsibility initiative: examining the influence of a collaborative service learning project on student learning. International Journal of Fashion Design, Technology and Education, 356-363. https://doi.org/10.1080/17543266.2019.1652854

In-COMAV (2020). Directrius. En In-COMAV: Innovació en Comunicació i Mitjans Audiovisuals. https://fima.ub.edu/grups/incomav/directrius.html

INE (Instituto Nacional de Estadística, 2018). Anuario estadístico de España. Madrid: INE. http://www.ine.es/prodyser/pubweb/anuario18/anu18_completo.pdf

Karabenick, S.A. \& Collins-Eaglin, J. (1997). Relation of perceived instructional goals and incentives to college students' use of learning strategies. Journal of Experimental Education, 65(4), 331-341.

Jenaro, C., Flores, N., Poy, R., González-Gil, F. \& Martín-Pastor, E. (2013). Metodologías docentes en la educación superior: percepciones del profesorado sobre su importancia y uso. Revista de Enseñanza Universitaria, 39, 1-16. http://institucional.us.es/revistas/universitaria/39/art_2.pdf

Martínez, M. (2009). Aprenentatge servei i construcció de ciutadania activa a la universitat: la dimensió social i cívica dels aprenentatges acadèmics a la universitat. En M. Martínez (ed.). Aprenentatge servei $i$ responsabilitat social de les universitats. Barcelona: Octaedro. https://aprenentatgeservei.cat/wp-content/uploads/biblio/180127_ApSUni.pdf

Mendía, R. (2012). El aprendizaje servicio como una estrategia inclusiva para superar las barreras al aprendizaje y a la participación. Educación Inclusiva, 5(1), 71-82.

Naciones Unidas (2015). Objetivos de desarrollo sostenible. Nueva York: Naciones Unidas. https://un.org/sustainabledevelopment/es 
Observatorio Audiovisual Europeo (2018). Yearbook 2017/2018, key trends. Television, cinema, video and on-demand audiovisual services: the pan-European picture. Estrasburgo: Consejo de Europa. https://rm.coe.int/yearbook-keytrends-2017-2018-en/16807b567e

Observatorio de Innovación Educativa (2016). Aprendizaje basado en retos. Monterrey: Instituto Tecnológico y de Estudios Superiores de Monterrey. https://observatorio.tec.mx/edutrendsabr

Peterson, C. \& Seligman, M.E.P. (2004). Character strengths and virtues: a handbook and classification. Nueva York: Oxford University Press/Washington, DC: American Psychological Association.

Puig, J.M., Batlle, R. Bosch, C. \& Palos, J. (2007). Aprendizaje servicio: educar para la ciudadanía. Barcelona: Octaedro.

Rodríguez, A. \& Tiana, A. (2015, junio-septiembre). Educación superior: mutación digital, Telos: cuadernos de comunicación e innovación, 101, 48-51.

Sánchez Serra, D. (2016). Location determinants of creative industries' firms in Spain. $\begin{array}{llrl}\text { Investigaciones } & \text { regionales, } & 34, & 23-48 .\end{array}$ https://old.aecr.org/images/ImatgesArticles/2016/5/02_SANCHEZi.pdf

Sobrado, L. \& Fernández, E. (2010). Competencias emprendedoras y desarrollo del espíritu empresarial en los centros educativos, Educación XXI, 13(1), 15-38.

Stenberg, R.J. (1998). A balance theory of wisdom. Review of General Psychology, 1, 347-365.

TERA (2014). The economic contribution of the creative industries to EU GDP and employment. http://teraconsultants.fr/en/issues/The-Economic-Contribution-of-the-Creative-Industries-to-EUin-GDP-and-Employment

Torre, S. \& Tejada, J. (2006). La dimensión emocional en la formación universitaria, Profesorado: revista de currículum y formación del profesorado, 10(2), 1-21.

Trigueros, C., Rivera, E. \& Torre, E. (2011). La evaluación en el aula universitaria: del examen tradicional a la autoevaluación. Revista Internacional de Medicina y Ciencias de la Actividad $\begin{array}{lllll}\text { Física } & y & \text { el } & \text { Deporte, } & \text { 12(47), }\end{array}$ http://cdeporte.rediris.es/revista/revista47/artevaluacion303.htm

Tubella, I. (2007). L'audiovisual a Catalunya, un motor en marxa? Barcelona: Pla Estratègic Metropolità de Barcelona.

Universidad Construye País (2006). Una manera de ser universidad: teoría y práctica en la experiencia chilena. Santiago de Chile: Proyecto Universidad Construye País.

Universidad de Barcelona (2019, 14 de febrero). Construyendo la Agenda 2030 de la UB. Universidad de Barcelona: noticias. https://ub.edu/web/ub/es/menu_eines/noticies/2019/02/028.html 


\section{AUTORES:}

\section{Jorge Franganillo}

Profesor e investigador. Universidad de Barcelona. Facultad de Información y Medios Audiovisuales.

franganillo@ub.edu

Índice H: 3 (Scopus), 10 (Google Scholar)

Orcid ID: https://orcid.org/0000-0003-4128-6546

Google Scholar: https://scholar.google.es/citations?user=SMpn1EcAAAAJ

\section{Lydia Sánchez}

Profesora e investigadora. Universidad de Barcelona. Facultad de Información y Medios Audiovisuales.

lsanchezg@ub.edu

Índice H: 1 (Scopus), 8 (Google Scholar)

Orcid ID: http://orcid.org/0000-0001-7814-0087

Google Scholar: https://scholar.google.es/citations?user=O9dj7XEAAAAJ

\section{María Ángeles García Asensio}

Profesora e investigadora. Universidad de Barcelona. Facultad de Filología y Comunicación.

garciaasensio@ub.edu

Índice H: 1 (Scopus), 6 (Google Scholar)

Orcid ID: https://orcid.org/0000-0002-5570-342X

Google Scholar: https://scholar.google.es/citations?user=Y3GQ_DoAAAAJ

\section{Anna Marquès}

Profesora e investigadora. Universidad de Barcelona. Facultad de Información y Medios Audiovisuales.

anna.marques@ub.edu

Índice H: 1 (Google Scholar)

Orcid ID: https://orcid.org/0000-0002-9964-9314 